\title{
Periodontal ligament proliferation and expressions of bone biomolecules upon orthodontic preloading: Clinical implications for tooth autotransplantation
}

\author{
Sasathorn Phutinart ${ }^{\mathrm{a}}$ (1) \\ Suttichai Krisanaprakornkit ${ }^{\mathrm{b}}$ \\ Anupong Makeudom ${ }^{\mathrm{c}}$ \\ Boonsiva Suzuki ${ }^{\mathrm{a}}$ \\ Eduardo Yugo Suzuki ${ }^{\mathrm{a}}$ (1)
}

${ }^{a}$ Department of Orthodontics, Faculty of Dentistry, Bangkokthonburi University, Bangkok, Thailand

${ }^{b}$ Department of Oral Biology and Diagnostic Sciences, Center of Excellence in Oral and Maxillofacial Biology, Faculty of Dentistry, Chiang Mai University, Chiang Mai, Thailand ${ }^{\mathrm{c}}$ Center of Excellence in Oral and Maxillofacial Biology, Faculty of Dentistry, Chiang Mai University, Chiang Mai, Thailand

\begin{abstract}
Objective: Preservation of the periodontal ligament (PDL) is vital to the success of tooth autotransplantation (TAT). Increased PDL volumes and facilitated tooth extraction have been observed upon orthodontic preloading. However, it is unclear whether any changes occur in the expressions of bone biomolecules in the increased PDL volumes. This study aimed to determine the expressions of runt-related transcription factor 2 (RUNX2), alkaline phosphatase (ALP), receptor activator of nuclear factor kappa-B ligand (RANKL), and osteoprotegerin (OPG) in PDL upon preloading. Methods: Seventy-two premolars from 18 patients were randomly assigned to experimental groups that received a leveling force for 1,2 , or 4 weeks or to a control unloaded group. Following extraction, PDL volumes from 32 premolars of eight patients $(21.0 \pm 3.8$ years $)$ were evaluated using toluidine blue staining. The expressions of the biomolecules in the PDL from 40 premolars of ten patients $(21.4 \pm 4.0$ years) were analyzed via immunoblotting. Results: The median percentage of stained PDL was significantly higher at 2 and 4 weeks after preloading than in the unloaded condition $(p<0.05)$. The median RUNX2 and ALP expression levels were significantly higher at 2 and 4 weeks after preloading than in the unloaded condition $(p<0.05)$, whereas the median RANKL/OPG ratios were significantly higher at 1 and 4 weeks after preloading $(p<0.05)$. Conclusions: Orthodontic preloading for 4 weeks enhances PDL volumes as well as the expressions of RUNX2, ALP and the RANKL/OPG ratio in the PDL, suggesting this loading period is suitable for successful TAT.
\end{abstract}

[Korean J Orthod 2020;50(3):188-196]

Key words: Bone biomolecules, Orthodontic preloading, Periodontal ligament, Tooth autotransplantation

Received December 30, 2019; Revised February 17, 2020; Accepted February 21, 2020.

Corresponding author: Eduardo Yugo Suzuki.

Director, Department of Orthodontics, Faculty of Dentistry, Bangkokthonburi University, 16/10 Leabklongtaweewatana Rd., Taweewatana District, Bangkok 10170, Thailand. Tel +66-53-274420 e-mail yugotmdu@hotmail.com

The present article has been presented and awarded at the 52nd Korean Association of Orthodontist Meeting.

How to cite this article: Phutinart S, Krisanaprakornkit S, Makeudom A, Suzuki B, Suzuki EY. Periodontal ligament proliferation and expressions of bone biomolecules upon orthodontic preloading: Clinical implications for tooth autotransplantation. Korean J Orthod 2020;50:188-196.

(C) 2020 The Korean Association of Orthodontists.

This is an Open Access article distributed under the terms of the Creative Commons Attribution Non-Commercial License (http://creativecommons.org/licenses/by-nc/4.0) which permits unrestricted non-commercial use, distribution, and reproduction in any medium, provided the original work is properly cited. 


\section{INTRODUCTION}

Preservation of the vital periodontal ligament (PDL) on a donor tooth is a key factor for successful tooth autotransplantation (TAT). ${ }^{1}$ The PDL attached to the root surface contains several cell types essential for the prevention of root resorption. ${ }^{2}$ PDL injuries often occur during the extraction of donor teeth, and this leads to root resorption and ankylosis, which result in unsuccessful TAT. ${ }^{3}$ The two main tissues involved in TAT healing are the PDL and alveolar bone. The healing of the PDL after TAT involves proliferative fibroblasts that are the most prevalent cell type within the PDL responsible for the production and alignment of collagen fiber bundles; in contrast, healing of the alveolar bone involves the bone remodeling process that begins with bone resorption by osteoclasts coupled with bone formation by osteoblasts. ${ }^{2}$ In addition to the synthesis and organization of collagen fibers, the proliferative PDL fibroblasts secrete various bone mediators that control osteoblast and osteoclast differentiation in response to mechanical stresses. ${ }^{4}$

Several bone mediators have been studied during orthodontic tooth movement, and their levels vary in the PDL of animals and humans. ${ }^{5-8}$ Among these mediators, runt-related transcription factor 2 (RUNX2) ) $^{5,9}$ and alkaline phosphatase (ALP) ${ }^{9-11}$ are commonly used to monitor osteoblast differentiation under mechanical stresses. RUNX2 is a specific transcription factor that activates mesenchymal cells of the bone marrow, leading to their differentiation into osteoblasts. Additionally, this transcription factor can regulate osteoblast maturation. ${ }^{6}$ ALP is commonly used to assess the phenotypic development of osteoblasts, ${ }^{12}$ and its expression reflects an early stage of osteoblastic differentiation. ${ }^{11}$ Osteoblasts also directly regulate osteoclast activity during bone remodeling. ${ }^{10}$ Osteoblastic lineage cells express receptor activator of nuclear factor kappa-B ligand (RANKL), ${ }^{13}$ which is a key factor mediating osteoclastogenesis and is considered an osteoclastic biomarker. ${ }^{10}$ RANKL triggers osteoclast formation and activity once it binds to its specific receptor, called RANK, on the osteoclast precursor cell membrane. To exert further control, osteoblasts produce a potent inhibitor of RANKL, i.e., osteoprotegerin (OPG), ${ }^{14}$ which is a member of the tumor necrosis factor receptor family. OPG functions by binding to RANKL and interrupting the RANKL-RANK interaction, thereby resulting in the inhibition of osteoclastogenesis. ${ }^{13}$

In a few previous studies, orthodontic force application before TAT resulted in increased PDL volume. ${ }^{15}$ Consequently, such force application is recommended to enhance the success rate of transplantation. ${ }^{16}$ However, the expressions of bone biomolecules within the PDL at different loading durations and the optimal preloading duration to obtain the greatest PDL volume remain unclear. Therefore, the aims of this study were to evaluate the optimal orthodontic preloading duration to increase PDL volume and to determine the expressions of the bone biomolecules RUNX2, ALP, RANKL, and OPG in human PDL tissue upon loading with a leveling force at different time points.

\section{MATERIALS AND METHODS}

\section{Patient selection}

Healthy, non-smoking patients requiring extraction of the first premolars for orthodontic treatment were recruited from the Postgraduate Clinic, Faculty of Dentistry, Bangkokthonburi University, Thailand, after obtaining written informed consent. The study protocol (\#9/2018) was approved by the Bangkokthonburi University $\mathrm{Hu}$ man Ethics Committee. The inclusion criteria were as follows: patients who had sound first premolars with complete root formation in their four quadrants without caries or restorations, and had good oral hygiene and a healthy periodontium. The exclusion criteria were as follows: patients with systemic infections or diseases who required medications, such as nonsteroidal inflammatory drugs, or those with severe crowding at the extraction sites.

Eighteen such patients undergoing orthodontic treatment were randomly divided into two groups for the measurement of PDL volume and determination of protein expression. For the measurement of PDL volume, 32 first premolars from eight patients (mean age, 21.0 \pm 3.8 years; six females and two males) were selected. The sample size of at least eight teeth for each period of loading was determined using G*Power software ver. 3.1.9.2 (Franz Faul; University of Kiel, Kiel, Germany), with an effect size $=1.45$ derived from the preliminary data, $\alpha=0.05$, and $1-\beta=0.95$. To determine protein expression, 40 first premolars from the ten remaining patients (mean age, $21.4 \pm 4.0$ years; eight females and two males) were selected. The sample size of at least nine teeth for each period of loading was determined with an effect size $=1.33$ derived from the preliminary data, $\alpha=0.05$, and $1-\beta=0.95$.

\section{Orthodontic preloading and tooth extraction}

The orthodontic leveling force was applied using a 0.016-inch continuous archwire, which is an improved superelastic nickel-titanium alloy wire (Sentalloy blue, Sentalloy ${ }^{\circledR}$; Tomy International Inc., Tokyo, Japan), inserted into a preadjusted edgewise bracket (Roth prescription; $0.018 \times 0.025$ inch, Tomy International Inc.) that was bonded on the first premolar and all remaining teeth. The bracket was attached using an indirect bonding technique to allow precise bracket positioning. ${ }^{17}$ For 
the first premolars, the brackets were passively placed to avoid either intrusive or extrusive movements. The wire possesses a shape-memory effect, which enables the generation of a light continuous force at 30-70 $\mathrm{g}$ in the oral cavity. ${ }^{18}$ The control unloaded premolars were extracted at each patient's first visit, whereas the experimental loaded premolars were extracted after orthodontic loading for 1, 2, or 4 weeks. Simple exodontia was performed by one oral surgeon, with the patients under local anesthesia induced using 4\% articaine and 1:100,000 epinephrine (Septanest SP ${ }^{\circledR}$; Septodont Co., Paris, France) administered via an infiltration technique (1.0 mL for the maxillary premolars) or an inferior alveolar nerve block together with lingual and long buccal nerve blocks ( $1.7 \mathrm{~mL}$ for the mandibular premolars). A conventional aspirating dental syringe and a disposable 30-gauge and 21-mm-long needle (Septoject $\mathrm{Xl}^{\circledR}$; Septodont Co.) were used for the infiltration, and a disposable 27-gauge and 30-mm-long needle (Septoject $\mathrm{XL}^{\circledR}$; Septodont Co.) were used for the nerve blocks. Both experimental and control premolars were atraumatically extracted by gentle separation of the gingiva using a straight elevator no. EL3S (Hu-Friedy Mfg. Co., LlC, Chicago, IL, USA), followed by the use of a no. 151 forceps (Hu-Friedy Presidential ${ }^{\circledR}$; Hu-Friedy Mfg. Co., LLC) to avoid injury to the PDL tissue. The extracted premolars were then washed with normal saline to remove blood and tissue debris.

\section{Assessment of periodontal ligament tissue}

Toluidine blue staining and determination of the remaining PDL volume on the root surface were performed using the protocol of Nakdilok et al. ${ }^{19}$ Briefly, the extracted teeth were stained with $0.04 \%(\mathrm{w} / \mathrm{v})$ toluidine blue (Sigma-Aldrich, St. Louis, MO, USA) and destained with phosphate buffered saline for 14 days. A stereomicroscope (Olympus SZX7; Olympus Corp., Tokyo, Japan) was used to observe all surfaces of the stained roots. A digitized image in a plane perpendicular to the tooth axis was then recorded using a charge-coupled device (Olympus E-330; Olympus Corp.) attached to the stereomicroscope. The ImageJ2 software (National Institutes of Health, Bethesda, MD, USA) was used to quantify the stained images, and the stained area in each image was determined as a percentage of the total area. The percentage of stained PDL was calculated using lmageJ2 by differentiating between the white and blue pixels, which represented the unstained and stained areas, respectively. The percentage of stained PDL of each tooth was then calculated.

\section{Expressions of bone biomolecules in periodontal ligament tissue}

The PDL tissue of the extracted premolars was scraped off from $3 \mathrm{~mm}$ below the cementoenamel junction to the root apex. Total protein was isolated by grinding the PDL tissue in a glass homogenizer using $350 \mu \mathrm{L}$ of lysis buffer from a NucleoSpin ${ }^{\circledR}$ RNA/Protein isolation kit (MACHEREY-NAGEL GmbH \& Co. KG, Düren, Germany) and $1 \%(\mathrm{v} / \mathrm{v}) \beta$-mercaptoethanol (Bio-Rad Laboratories, Inc., Hercules, CA, USA). The lysate was transferred to a 1.5-mL microcentrifuge tube and stored at $-80^{\circ} \mathrm{C}$ until simultaneous western blot analysis of every sample from the ten patients. The frozen lysates were thawed and their total protein was extracted using the NucleoSpin ${ }^{\circledR}$ RNA/Protein isolation kit (MACHEREY-NAGEL GmbH \&t Co. KG) following the manufacturer's instruction. In brief, the lysates were first centrifuged at $1,100 \times g$ for 1 minute to remove tissue debris by using filter tubes, and the filtrated lysates were mixed with 70\% ethanol at an equal volume. The mixtures were centrifuged using the NucleoSpin ${ }^{\circledast}$ columns to collect their protein fraction from the flow-through solution. Protein in the solution was precipitated and dissolved in a protein solving buffer containing the reducing agent Tris (2-carboxyethyl) phosphine hydrochloride. The quantity of total protein in each sample was determined using ultraviolet absorbance at $280 \mathrm{~nm}$ in a NanoDrop ${ }^{\mathrm{TM}} 2000$ spectrophotometer (Thermo Scientific ${ }^{\mathrm{TM}}$, Rockford, IL, USA). A $20-\mu$ g quantity of total protein was resolved using 10\% sodium dodecyl sulfate polyacrylamide gel electrophoresis under an electrical current at $100 \mathrm{~V} / 300$ W for 135 minutes, and was transferred to nitrocellulose membranes under an electrical current at $100 \mathrm{~mA} / 300$ $\mathrm{W}$ for 12 hours. To block non-specific binding, the membranes were incubated for 1 hour in 5\% (w/v) nonfat dry milk (Santa Cruz Biotechnology lnc., Santa Cruz, CA, USA) in $0.1 \%(\mathrm{v} / \mathrm{v})$ Tween-20/Tris-buffered saline. Subsequently, the membranes were incubated overnight at $4^{\circ} \mathrm{C}$ with the primary antibodies. On the following day, the membranes were washed and incubated with the secondary antibodies for 1 hour. The primary and secondary antibodies used in this study are summarized in Table 1. After washing, the membranes were reacted with LumiGLO Reserve ${ }^{\mathrm{TM}}$ Chemiluminescent reagent (KPL, Gaithersburg, MD, USA). Chemiluminescent signals were captured using the ChemiDoc XRS gel documentation system (Bio-Rad Laboratories, Inc.). The intensities of RUNX2, ALP, RANKL, and OPG bands at their predicted size were measured using ImageJ2 and normalized by that of the beta actin band in each sample. Thereafter, the normalized intensities of these proteins were compared between the experimental and control groups. In addition, the relative ratios of normalized RANKL to OPG were determined and compared between the experimental and control groups. 
Table 1. List of antibodies

\begin{tabular}{|c|c|c|c|}
\hline Antibody & Donor & Dilution & Supplier \\
\hline \multicolumn{4}{|l|}{ Primary antibody } \\
\hline Polyclonal IgG anti-human RUNX2/CBFA1 & Goat & $1: 2,000$ & R\&D systems, Minneapolis, MN, USA \\
\hline 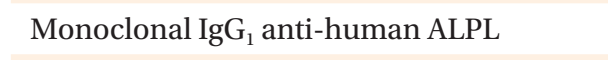 & Mouse & $1: 2,000$ & $\mathrm{R} \& \mathrm{D}$ systems \\
\hline 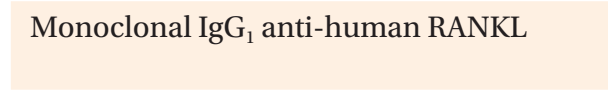 & Mouse & $1: 500$ & $\begin{array}{l}\text { Santa Cruz Biotechnology, } \\
\text { Santa Cruz, CA, USA }\end{array}$ \\
\hline Monoclonal $\mathrm{IgG}_{1}$ anti-human OPG & Mouse & $1: 500$ & Santa Cruz Biotechnology \\
\hline 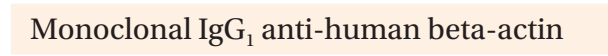 & Mouse & $1: 1,000$ & Santa Cruz Biotechnology \\
\hline \multicolumn{4}{|l|}{ Secondary antibody } \\
\hline Anti-rabbit immunoglobulins & $\begin{array}{l}\text { Horseradish peroxidase (HRP)- } \\
\text { conjugated swine }\end{array}$ & $1: 2,000$ & Dako, Glostrup, Denmark \\
\hline Anti-mouse immunoglobulins & HRP-conjugated rabbit & $1: 2,000$ & R\&D systems \\
\hline Anti-goat IgG (H + L) & HRP-mouse & $1: 2,000$ & Thermo Scientific, Rockford, IL, USA \\
\hline
\end{tabular}

IgG, Immunoglobulin G; RUNX2, runt-related transcription factor 2; CBFA1, core-binding factor subunit alpha-1; ALPL, alkaline phosphatase; RANKL, receptor activator of nuclear factor kappa-B ligand; OPG, osteoprotegerin; $\mathrm{H}$, heavy chain; L, light chain.

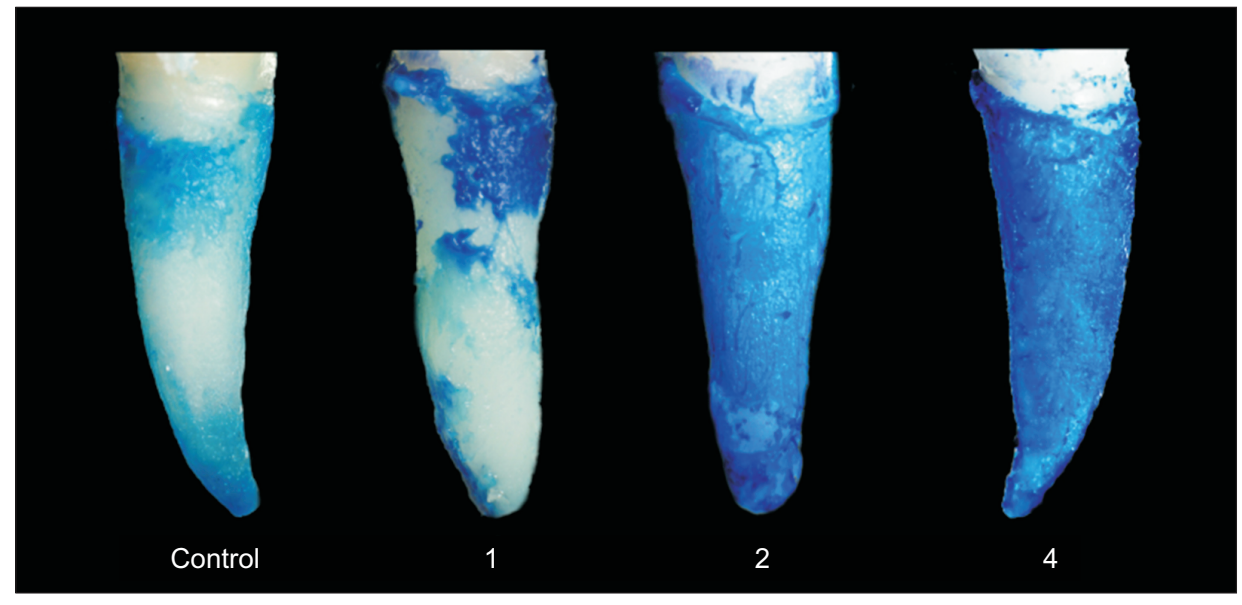

Figure 1. Representative image illustrating the stained periodontal ligament in an unloaded tooth (control) and in teeth after orthodontic loading for 1, 2, or 4 weeks.

\section{Statistical analysis}

Data were analyzed using IBM SPSS Statistics for Windows, version 19.0 (IBM Corp., Armonk, NY, USA). Comparisons between the percentages of overall stained PDL tissue at different time points, significant differences between protein expressions, and the RANKL/OPG ratios at different orthodontic loading durations were tested using the Friedman test, followed by the Wilcoxon signedrank test. The differences were considered statistically significant if $p$-values were less than 0.05. Spearman's method was used to analyze correlations between the percentages of stained PDL, protein expressions, or the RANKL/OPG ratios and increased loading durations.

\section{RESULTS}

Enhancement of periodontal ligament tissue volume upon orthodontic preloading

Larger areas of stained PDL were observed on the root surface of the loaded teeth, particularly those loaded for 2 and 4 weeks, than on those of the control unloaded teeth (Figure 1). The median percentages of stained PDL were significantly greater in the teeth loaded for 2 and 4 weeks than in the control unloaded teeth and in the teeth loaded for 1 week ( $p<0.05$; Figure 2$)$. In addition, a strong positive correlation was found between the percentage of stained PDL and increased preloading durations $(r=0.608, p<0.001)$. 
Significant increases in the expressions of RUNX2 and ALP and the RANKL/OPG ratio upon orthodontic preloading

The expressions of RUNX2 and ALP were more enhanced in the premolars loaded for 1, 2, and 4 weeks

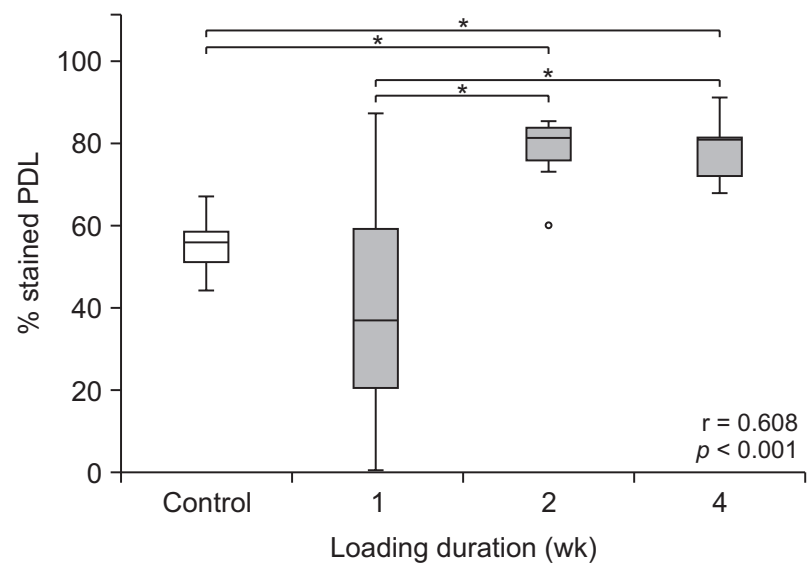

Figure 2. Percentage of stained periodontal ligament (PDL) in unloaded teeth compared to that in loaded teeth for different loading durations. ${ }^{*} p<0.05$.
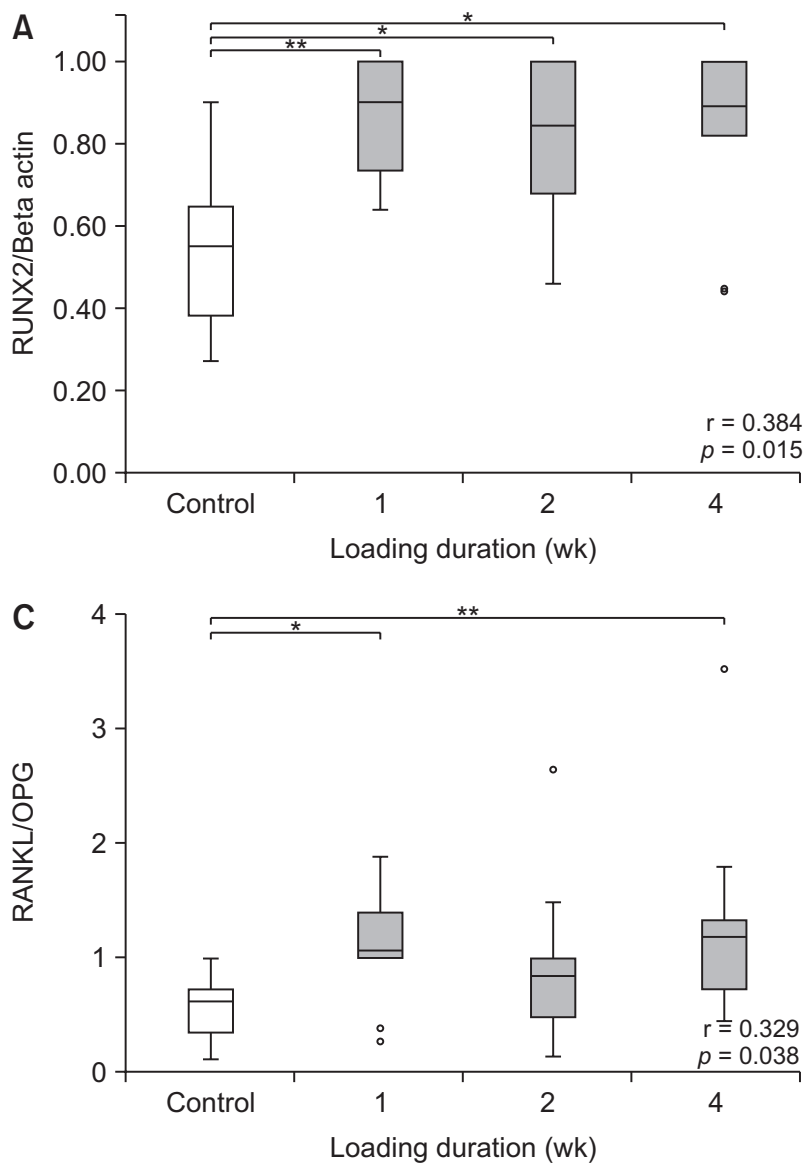

than in the control unloaded teeth, whereas those of RANKL and OPG varied among the unloaded and loaded teeth over different preloading durations (Figure 3). The expression of beta actin was approximately equal among the different samples within each patient. Densitometry revealed that the normalized median expressions

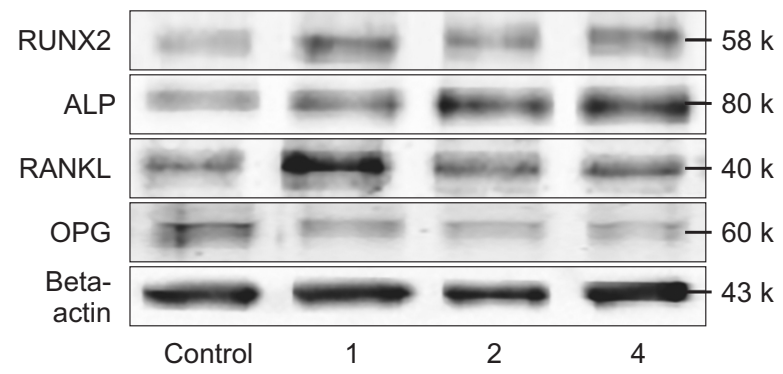

Figure 3. Representative immunoblotting results of the expressions of runt-related transcription factor 2 (RUNX2), alkaline phosphatase (ALP), receptor activator of nuclear factor kappa-B ligand (RANKL), osteoprotegerin (OPG), and beta actin in human periodontal ligament tissues after orthodontic loading for 1, 2, or 4 weeks, or unloaded as a control.

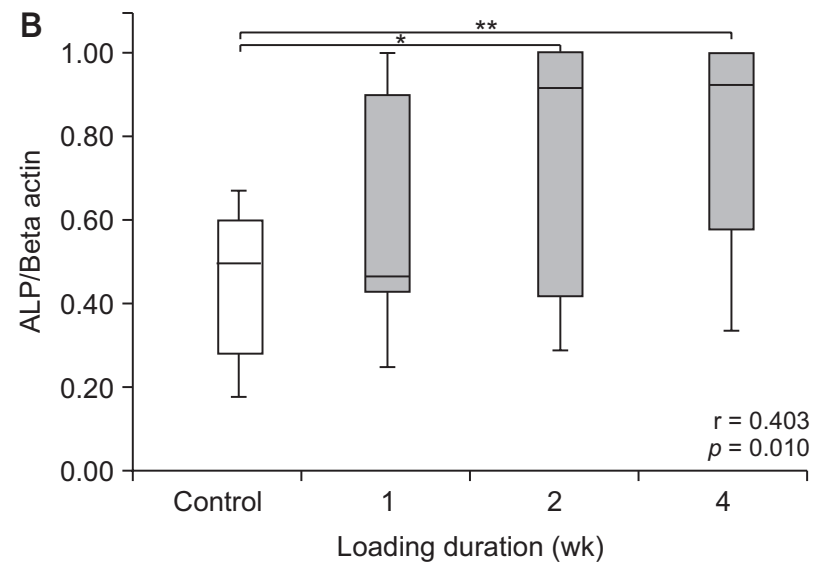

Figure 4. Box plot diagrams showing significantly enhanced expressions of runt-related transcription factor 2 (RUNX2) (A) and alkaline phosphatase (ALP) (B) normalized by the expression of beta actin, as well as the receptor activator of nuclear factor kappa-B ligand (RANKL)/ osteoprotegerin (OPG) ratio (C), at different loading durations (gray boxes) compared to those of controls that are unloaded (empty boxes). Moderately positive correlations are found between the expressions of RUNX2 (A) and ALP (B) or the RANKL/OPG ratio (C) and loading durations from 1 to 4 weeks. The horizontal line within each box represents the median. ${ }^{*} p<0.05 ;{ }^{* *} p<0.01$. 
of RUNX2 and ALP were significantly greater in the premolars loaded for 2 and 4 weeks than in the control unloaded premolars (Figure 4A and 4B). Moreover, moderately positive correlations were found between the normalized expression of RUNX2 or ALP and increased preloading durations $(r=0.384, p=0.015$ and $r=0.403$, $p=0.010$, respectively). By contrast, no difference was observed in the normalized median expression of RANKL or OPG between the loaded and unloaded premolars (data not shown). However, the median RANKL/OPG ratios were significantly higher in the premolars loaded for 1 and 4 weeks than in the unloaded premolars $(p<0.05$; Figure 4C). Furthermore, the RANKL/OPG ratio positively correlated with increased preloading durations $(\mathrm{r}=0.329$, $p=0.038$ ).

\section{DISCUSSION}

PDL is a fiber-reinforced tissue containing various types of cells that play important roles in the response to mechanical stresses, maintaining the periodontium, and promoting periodontal regeneration. ${ }^{9}$ The results of this study demonstrated that orthodontic preloading led to macroscopic and molecular alterations in the PDL tissue. During the early stage of this process, i.e., after the application of the leveling force for 1 week, no significant changes were observed in the percentages of stained PDL, whereas significant increases in RUNX2 expression and the RANKL/OPG ratio were noted. Two weeks after orthodontic preloading, PDL volume and the expression of ALP were significantly increased, and the degree of expression of RUNX2 remained high. At the end of the observation, i.e., after 4 weeks of preloading with the leveling force, PDL volume and the expressions of RUNX2 and ALP along with the RANKL/OPG ratio were approximately at their maximal levels and were greater in the loaded teeth than in the unloaded teeth. Taken together, the preloading duration appears to be a crucial factor that increases proliferative PDL volumes, as shown by an increase in the percentage of stained PDL, the expressions of RUNX2 and ALP, as well as the RANKL/OPG ratio within the PDL.

Preapplication of orthodontic loading for various durations has been previously performed in several studies. In an animal study, the preloading of a light continuous orthodontic force for 7 days resulted in rich PDL tissue attached to the extracted teeth. ${ }^{15} \mathrm{~A}$ study performed in humans also demonstrated that preoperative application of an extrusion force for 2 to 3 weeks helped prevent extraction failure and increased the success rate of tooth replantation. ${ }^{16}$ Moreover, our previous study showed a significant increase in PDL volume and facilitated tooth extraction upon orthodontic preloading for 4 weeks. ${ }^{19}$ However, the time-course study of orthodontic preload- ing that may help increase the success rate of TAT has not been discussed.

Our study suggests that increased proliferative PDL and the expression profiles of the four bone biomolecules derived from the proliferative PDL are associated with various clinical phases of orthodontic tooth movement. In the initial phase, PDL becomes disorganized; the collagen fiber orientation in the PDL is considerably changed by the function of PDL fibroblasts. ${ }^{20}$ These cells play crucial roles in synthesizing new collagen fibers and ground substances while simultaneously breaking down old connective tissue in the PDL. ${ }^{21}$ Therefore, no significant changes in net PDL volumes were observed at the first week, which corresponded to no change in the median percentage of stained PDL (Figure 2). However, at the molecular level, the mechanosensitive PDL cells can convert mechanical stimuli from the orthodontic preloading force into biological signals, which regulate osteoblastogenesis ${ }^{22}$ and osteoclastogenesis. ${ }^{23}$ These are consistent with significant increases in the expression of RUNX2 and the RANKL/OPG ratio, respectively, at the first week. The upregulated expression of RUNX2 indicates an induction of mesenchymal cells into differentiated osteoblasts. ${ }^{5}$ On the contrary, an increase in the RANKL/OPG ratio, either from the enhanced expression of RANKL due to inflammation within the PDL caused by orthodontic preloading, ${ }^{8,13}$ from the decreased expression of OPG in the PDL, ${ }^{8,13}$ or both, probably accounts for the significantly increased RANKL/OPG ratio observed at the first week after orthodontic preloading, and would lead to enhanced osteoclastogenesis and subsequent alveolar bone resorption during the initial phase. $^{24}$

Subsequently, the lag and post-lag phases involve reorganization of the fibrous attachment apparatus by augmented production of new periodontal fibrils, which takes place throughout the PDL. ${ }^{20}$ Consequently, the enhanced synthesis of PDL fibers would be in line with significant increases in the percentage of stained PDL observed at 2 and 4 weeks after orthodontic preloading. In addition, the significant increases in the expressions of RUNX2 and ALP in the PDL observed at 2 and 4 weeks after orthodontic preloading would help recruit and activate new osteoblast progenitors and quiescent bone lining cells to commence the production of new bone matrix..$^{25}$ After 4 weeks of orthodontic force application, continuous PDL cell division, resulting in continual collagen synthesis, ${ }^{26}$ and high capacities of PDL and alveolar bone remodeling ${ }^{27}$ are substantiated by significant and continuing enhancement of PDL volume and the expressions of RUNX2 and ALP as well as the RANKL/OPG ratio.

The kinetic profiles of the induced expressions of RUNX2 and ALP and the increased RANKL/OPG ratio in 
human PDL tissue upon receiving the leveling force observed in this study are in agreement with the findings of previous in vivo studies that have shown upregulated expression of RUNX2, ${ }^{28}$ raised ALP levels, ${ }^{29}$ elevated gingival crevicular fluids (GCF) and salivary RANKL levels, and decreased GCF and salivary OPG levels ${ }^{8,30}$ upon orthodontic preloading in a time-dependent manner. However, notably, no difference was observed in the expression of RANKL or OPG among the different loading durations (data not shown). This may be attributable to the first observation period at 1 week that may already have been too late to detect any initial changes in the levels of RANKL or OPG, because they have been previously reported to be significantly elevated or lowered, respectively, upon orthodontic loading for 24 hours in the human GCF. ${ }^{8}$

This study reveals that orthodontic preloading induces alterations at the macroscopic level, as evidenced by the significant increase in the proliferative PDL tissue, which may help prevent the occurrence of denuded root surfaces due to tooth extraction, possibly leading to a reduction in ankylosis and root resorption after TAT. In addition, orthodontic preloading aids in facilitated tooth extraction, ${ }^{19}$ which would decrease the possibility of root fracture of the donor tooth. Moreover, at the molecular level, orthodontic preloading results in significant increases in the expressions of RUNX2 and ALP that are essential for osteoblast differentiation and new bone formation and in the RANKL/OPG ratio that induces bone resorption. All of these molecular changes that affect bone formation and resorption would facilitate adaptation of the donor tooth to the alveolar bone at the recipient site, and promote bone remodeling during the healing of transplanted teeth. Therefore, application of orthodontic preloading results in both PDL proliferation and increased expressions of bone-related molecules. Increased PDL volume ensures easy and atraumatic extraction and provides thick PDL coverage around the

Table 2. Outcomes of orthodontic preloading compared to those of unloading

\begin{tabular}{lccc}
\hline \multirow{2}{*}{ Outcome } & \multicolumn{3}{c}{ Loading duration (wk) } \\
\cline { 2 - 4 } & $\mathbf{1}$ & $\mathbf{2}$ & $\mathbf{4}$ \\
\hline Enhancement of PDL tissue & NS & $*$ & $*$ \\
RUNX2 & $* *$ & $*$ & $*$ \\
ALP & NS & $*$ & $* *$ \\
RANKL/OPG ratio & $*$ & NS & $* *$ \\
\hline
\end{tabular}

PDL, Periodontal ligament; RUNX2, runt-related transcription factor 2; ALP, alkaline phosphatase; RANKL, receptor activator of nuclear factor kappa-B ligand; OPG, osteoprotegerin; NS, no significant.

${ }^{*} p<0.05 ;{ }^{* *} p<0.01$. radicular portion, which is beneficial for reducing ankylosis risks. Furthermore, increased expressions of bonerelated molecules, which are involved in bone remodeling, would modulate the bone healing response. These beneficial effects highlight the clinical implications of orthodontic preloading for good PDL and bone healing, which lead to increased TAT success rate. However, further clinical studies are necessary to investigate the overall benefits of orthodontic preloading on TAT.

Although this study showed no differences in PDL volumes and the expressions of bone biomolecules between 2 and 4 weeks, the optimal orthodontic preloading duration to gain benefits in both PDL volume and bone tissue responses probably is 4 weeks, when all the tested parameters were significantly higher than those in the controls (Table 2). Additionally, this study is the first to show not only the enhancement of PDL volumes but also the time-course changes in the expressions of important bone biomolecules within human PDL tissues upon orthodontic preloading using a leveling force. Moreover, our investigations on protein expressions were performed in human PDL tissues rather than in the GCF or saliva, as previously reported. . $^{8,30}$

In this study, a continuous leveling force was used to determine the effect of orthodontic preloading on PDL volumes as well as the expressions of bone biomolecules within the PDL. Despite the leveling force used to generate jiggling movement for the first premolars, the expressions of bone biomolecules likely vary between the pressure and tension sides. This issue was not, however, addressed in this study owing to the collection of total proteins from the PDL tissue of the whole root surface. Consequently, our results should be cautiously interpreted as the total effect of PDL responses upon orthodontic preloading, and not as a specific effect at the pressure or tension side. Other factors, such as the magnitudes and types of force, may differently influence the biological responses of the PDL tissue. Therefore, it would be interesting to further investigate the effect of these factors on the PDL. Moreover, future long-term clinical research is essential to confirm the success rate of TAT after the application of an orthodontic preloading force on the donor tooth for 4 weeks.

\section{CONCLUSION}

The findings of this study demonstrated that orthodontic preloading for 4 weeks significantly increased proliferative PDL tissue, the expressions of RUNX2 and ALP, and the RANKL/OPG ratio, which may be beneficial for improving the success rate of TAT. 


\section{CONFLICTS OF INTEREST}

No potential conflict of interest relevant to this article was reported.

\section{ACKNOWLEDGEMENTS}

This study was financially supported by Bangkokthonburi University to S.P., Chiang Mai University, and the Thailand Research Fund grants (\#BRG6080001, \#RDG5750069, and \#MRG5080347) to S.K., B.S., and E.Y.S., respectively.

We thank Dr. Thanapat Sastraruji for his assistance in statistical analysis. We would also like to express our gratitude to Dr. M. Kevin 0 Carroll for his critical reading of the manuscript.

\section{REFERENCES}

1. Andreasen JO. Periodontal healing after replantation and autotransplantation of incisors in monkeys. Int J Oral Surg 1981;10:54-61.

2. Tsukiboshi M, Andreasen J0. Autotransplantation of teeth. Chicago: Quintessence Pub Co; 2001.

3. Sugai T, Yoshizawa M, Kobayashi T, Ono K, Takagi $\mathrm{R}$, Kitamura $\mathrm{N}$, et al. Clinical study on prognostic factors for autotransplantation of teeth with complete root formation. Int J Oral Maxillofac Surg 2010;39:1193-203.

4. Li M, Zhang C, Yang Y. Effects of mechanical forces on osteogenesis and osteoclastogenesis in human periodontal ligament fibroblasts: a systematic review of in vitro studies. Bone Joint Res 2019;8:19-31.

5. Brooks PJ, Nilforoushan D, Manolson MF, Simmons CA, Gong SG. Molecular markers of early orthodontic tooth movement. Angle Orthod 2009;79:110813.

6. Li B, Zhang YH, Wang LX, Li X, Zhang XD. Expression of OPG, RANKL, and RUNX2 in rabbit periodontium under orthodontic force. Genet Mol Res 2015;14:19382-8.

7. Garlet TP, Coelho U, Silva JS, Garlet GP. Cytokine expression pattern in compression and tension sides of the periodontal ligament during orthodontic tooth movement in humans. Eur J Oral Sci 2007; 115:355-62.

8. Nishijima Y, Yamaguchi M, Kojima T, Aihara N, Nakajima R, Kasai K. Levels of RANKL and OPG in gingival crevicular fluid during orthodontic tooth movement and effect of compression force on releases from periodontal ligament cells in vitro. Orthod Craniofac Res 2006;9:63-70.

9. Zhang L, Liu W, Zhao J, Ma X, Shen L, Zhang Y, et al. Mechanical stress regulates osteogenic differenti- ation and RANKL/OPG ratio in periodontal ligament stem cells by the $\mathrm{Wnt} / \beta$-catenin pathway. Biochim Biophys Acta 2016;1860:2211-9.

10. Tripuwabhrut P, Mustafa M, Gjerde CG, Brudvik P, Mustafa K. Effect of compressive force on human osteoblast-like cells and bone remodelling: an in vitro study. Arch Oral Biol 2013;58:826-36.

11. Pavlin D, Dove SB, Zadro R, Gluhak-Heinrich J. Mechanical loading stimulates differentiation of periodontal osteoblasts in a mouse osteoinduction model: effect on type 1 collagen and alkaline phosphatase genes. Calcif Tissue Int 2000;67:163-72.

12. Banerjee C, McCabe LR, Choi JY, Hiebert SW, Stein JL, Stein GS, et al. Runt homology domain proteins in osteoblast differentiation: AML3/CBFA1 is a major component of a bone-specific complex. J Cell Biochem 1997;66:1-8.

13. Yamaguchi M. RANK/RANKL/OPG during orthodontic tooth movement. Orthod Craniofac Res 2009;12:113-9.

14. Kapasa ER, Giannoudis PV, Jia X, Hatton PV, Yang $X B$. The effect of RANKL/OPG balance on reducing implant complications. J Funct Biomater 2017;8:E42.

15. Suzaki Y, Matsumoto Y, Kanno Z, Soma K. Preapplication of orthodontic forces to the donor teeth affects periodontal healing of transplanted teeth. Angle Orthod 2008;78:495-501.

16. Choi YH, Bae JH, Kim YK, Kim HY, Kim SK, Cho BH. Clinical outcome of intentional replantation with preoperative orthodontic extrusion: a retrospective study. Int Endod J 2014;47:1168-76.

17. Suzuki EY, Suzuki B. Guiding template for direct bracket placement: a hybrid technique. Austin J Dent 2019;6:1127.

18. Miura F, Mogi M, Ohura Y, Hamanaka H. The superelastic property of the Japanese NiTi alloy wire for use in orthodontics. Am J Orthod Dentofacial Orthop 1986;90:1-10.

19. Nakdilok K, Langsa-ard S, Krisanaprakornkit S, Suzuki EY, Suzuki B. Enhancement of human periodontal ligament by preapplication of orthodontic loading. Am J Orthod Dentofacial Orthop 2020;157:186-93.

20. Thilander B. Tissue reactions in orthodontics. In: Graber LW, Vanarsdall RL, Vig KWL, eds. Orthodontics: current principles and techniques. 5th ed. Philadelphia: Elsevier/Mosby; 2011. p. 247-86.

21. Ten Cate AR, Deporter DA, Freeman E. The role of fibroblasts in the remodeling of periodontal ligament during physiologic tooth movement. Am J Orthod 1976;69:155-68.

22. Shen T, Qiu L, Chang H, Yang Y, Jian C, Xiong J, et al. Cyclic tension promotes osteogenic differentiation in human periodontal ligament stem cells. Int $\mathrm{J}$ 
Clin Exp Pathol 2014;7:7872-80.

23. Kanzaki H, Chiba M, Shimizu Y, Mitani H. Periodontal ligament cells under mechanical stress induce osteoclastogenesis by receptor activator of nuclear factor kappaB ligand up-regulation via prostaglandin E2 synthesis. J Bone Miner Res 2002;17:210-20.

24. Dunn MD, Park CH, Kostenuik PJ, Kapila S, Giannobile WV. Local delivery of osteoprotegerin inhibits mechanically mediated bone modeling in orthodontic tooth movement. Bone 2007;41:446-55.

25. Krishnan V, Davidovitch Z. Cellular, molecular, and tissue-level reactions to orthodontic force. Am J Orthod Dentofacial Orthop 2006;129:469.e1-32.

26. Bumann A, Carvalho RS, Schwarzer CL, Yen EH. Collagen synthesis from human PDL cells following orthodontic tooth movement. Eur J Orthod 1997;19:29-37.
27. Pilon JJ, Kuijpers-Jagtman AM, Maltha JC. Magnitude of orthodontic forces and rate of bodily tooth movement. An experimental study. Am J Orthod Dentofacial Orthop 1996;110:16-23.

28. Han J, Xu X, Zhang B, Chen B, Hang W. Expression of ATF4 and RUNX2 in periodontal tissue of pressure side during orthodontic tooth movement in rat. Int J Clin Exp Med 2015;8:934-8.

29. Insoft M, King GJ, Keeling SD. The measurement of acid and alkaline phosphatase in gingival crevicular fluid during orthodontic tooth movement. Am J Orthod Dentofacial Orthop 1996;109:287-96.

30. Flórez-Moreno GA, Isaza-Guzmán DM, TobónArroyave Sl. Time-related changes in salivary levels of the osteotropic factors sRANKL and OPG through orthodontic tooth movement. Am J Orthod Dentofacial Orthop 2013;143:92-100. 